\title{
CrimRxiv
}

\section{Crime, justice and criminology in the Republic of Ireland}

Claire Hamilton

Published on: Mar 02, 2022

DOI: 10.21428/cb6ab371.27c48de6

License: Creative Commons Attribution 4.0 International License (CC-BY 4.0). 
